\title{
Ectopic expression of systemic RNA interference defective protein (SID-1) in embryonic stem cells: Implications for high-throughput gene screening
}

\author{
Jennifer C Moore ${ }^{1,2, *}$, Suk Ying Tsang ${ }^{1,2, *}$, Rika Van Huizen ${ }^{1,2}$, Camie WY Chan ${ }^{2,3}$, Ronald A Li ${ }^{1,2,3}$ \\ ${ }^{1}$ Stem Cell Program and ${ }^{2}$ Department of Cell Biology \& Human Anatomy, University of California, Davis, CA, USA; ${ }^{3}$ Institute of \\ Pediatric Regenerative Medicine, Shriners Hospital for Children of North America, Sacramento, CA, USA
}

RNA interference (RNAi), a post-transcriptional gene silencing mechanism originally described in C. elegans, involves sequence-specific mRNA degradation mediated by double-stranded RNAs (dsRNAs). Passive dsRNA uptake has been uniquely observed in $C$. elegans due to the expression of systemic RNA interference defective-1 (SID-1). Here we investigated the ability of ectopic SID-1 expression in pluripotent embryonic stem cells (ESCs) to enable passive cellular uptake of dsRNAs. Reporter firefly luciferase (luc ${ }^{\text {Fir }}$ ) and either GFP or SID-1-GFP were co-expressed in ESCs, followed by simple soaking in control (non-silencing or Renilla luciferasespecific, luc ${ }^{\text {Ren }}$ ) or luc ${ }^{\text {Fir }}$-specific dsRNAs (100bp and 500bp). Soaking of SID-1-GFP- but not GFP-expressing ESCs in luc ${ }^{\text {Fir }}$-dsRNAs suppressed luc ${ }^{\text {Fir }}$ activity. By contrast, suppression was not observed without lucFirdsRNAs, or when either GFP- or SID-1-GFPexpressing cells were soaked in control dsRNAs. These results may lead to high-throughput experimental strategies for studying ESC differentiation and novel approaches to genetically inhibit or eliminate the tumorigenicity of ESCs.

Keywords: embryonic stem cells, C. elegans, RNA interference, RNA interference defective-1

Cell Research (2008) 18:s129. doi: 10.1038/cr.2008.219; published online 4 August 2008

*These two authors contributed equally to this work.

Correspondence: Ronald Li

E-mail: ronaldli@ucdavis.edu 